\title{
ON THE SECOND FUNDAMENTAL THEOREM OF NEVANLINNA
}

\author{
ARTURO FERNANDEZ ARIAS
}

\begin{abstract}
It is shown that a condition on the size of the exceptional set in the second fundamental theorem of Nevanlinna cannot be improved. The method is based on a construction of Hayman and also makes use of a quantitative version of a result of $F$. Nevanlinna about the growth of the characteristic function of a meromorophic function omitting a finite number of points
\end{abstract}

1. Introduction. The second fundamental theorem of Nevanlinna about the value distribution of meromorphic functions states that for a meromorphic function $F$ in $|z| \leq r$ and $q>2$ distinct values $a_{1}, a_{2}, \ldots, a_{q}$ of the complex extended plane we have the inequality

$$
(q-2) T(r, F)<\sum_{\nu=1}^{q} N\left(r, a_{\nu}\right)-N_{1}(r)+S(r, F),
$$

where $N_{1}(r)$ is positive and $S(r, F)$ is given by

$$
\begin{aligned}
S(r, F)= & m\left(r, \frac{F^{\prime}}{F}\right)+m\left\{r, \sum_{\nu=1}^{q} \frac{F^{\prime}}{F-a_{\nu}}\right\}+q \log ^{+} \frac{3 q}{\delta} \\
& +\log 2+\log \frac{1}{\left|F^{\prime}(0)\right|} \text { if }\left|a_{\mu}-a_{\nu}\right| \geq \delta \text { for } 1 \leq \mu<\nu \leq q,
\end{aligned}
$$

with modifications if $F(0)=\infty$ or $F^{\prime}(0)=0$.

The quantity $S(r, F)$ will be, in general, negligible with respect to $T(r, F)$. More precisely

$$
S(r, F)=O\{\log T(r, F)\}+O\{\log r\}
$$

as $r \rightarrow \infty$ through all values if $F$ has finite order, and outside an exceptional set of finite measure otherwise.

In particular (1.2) implies

$$
S(r, F)=o(T(r, F))
$$

for a transcendental function $F$ outside an exceptional set $E_{1}$ of finite measure.

From (1.1) and (1.3) we obtain

$$
(q-2) T(r, F)<\left(\sum_{\nu=1}^{q} N\left(r, a_{\nu}\right)\right)(1+o(1)) .
$$

Received by the editors January 15,1986 .

1980 Mathematics Subject Classification (1985 Revision). Primary 30D35.

Key words and phrases. Characteristic function, zeros, poles, distribution, growth. 
But we could still have the relation (1.4) even if (1.3) fails. We can then consider the exceptional set $E_{2}$ outside which (1.4) is true. From what we have said above we deduce that we can choose

$$
E_{1}, E_{2} \text { so that } E_{2} \subset E_{1} .
$$

In [1] some conditions on the size of $E_{1}$ were given; in particular, it was shown that we can take $E_{1}$ independent of $\lambda$ so that

$$
\int_{E_{1}} r^{\lambda} d r<\infty \text { for every } \lambda \geq 0 .
$$

It is clear from (1.5) that we can also choose $E_{2}$ independent of $\lambda$ so that

$$
\int_{E_{2}} r^{\lambda} d r \leq \int_{E_{1}} r^{\lambda} d r<\infty \text { for every } \lambda \geq 0 .
$$

In this paper we show that this condition is the best that one can obtain in this direction for the set $E_{2}$.

2. Statement of the main result. Precisely we shall prove

THEOREM 1. For any function $\Phi(r)$ such that

$$
\Phi(r) / r^{\lambda} \rightarrow \infty \text { for every } \lambda \geq 0,
$$

there exists an entire function such that the corresponding exceptional set $E_{2}$ satisfies

$$
\int_{E_{2}} \Phi(r) d r=\infty
$$

The proof of this result is based on a construction of Hayman [5]. The function $f$ shown is defined so that it approximates a sequence of polynomials with few zeros and $i$-values on a sequence of corresponding expanding circles. In this way $N(r, 0), N(r, i)$ will not grow very quickly for the function $f$ either.

We shall use a result due to F. Nevanlinna (see R. Nevanlinna [8]) to show that all the auxiliary functions in [5] have characteristic growing near $r=1$ quicker than

$$
C \log \frac{1}{1-r} \text { for certain constant } C \text { 's. }
$$

Using this fact we shall find precise estimates of the size of the set where

$$
N(r, 0)+N(r, i)+N(r, \infty)<\frac{1}{2} T(r, f),
$$

and check that this set is big enough to satisfy (2.2).

\section{Some preliminary results.}

3.1. In the proof of Theorem 1 we shall use several auxiliary results. First of all we shall prove the above-mentioned result of F. Nevanlinna but we shall obtain precise quantitative values for the constants involved, whereas he only obtained a quantitative result. 
LEMMA 3.1. Suppose that $a_{1}, a_{2}, \ldots, a_{q-1}$ are distinct complex numbers such that for a certain $\delta$, with $1>\delta>0$,

$$
\left|a_{\mu}-a_{\nu}\right| \geq \delta, \quad a_{\mu} \leq \frac{1}{\delta}, \quad 1 \leq \mu<\nu \leq q-1,
$$

and assume that $F$ maps the unit disc conformally onto the universal covering surface over the Riemann sphere punctured at $a_{1}, \ldots, a_{q-1, \infty}$, satisfying

$$
\left|F(0)-a_{\mu}\right| \geq \delta, \quad|F(0)| \leq \frac{1}{\delta}, \quad \mu=1, \ldots, q-1
$$

Then

$$
T(r, F) \geq C_{1}(q) \log \frac{1}{1-r}, \quad 1-A_{0}\left(\frac{1}{2} \delta^{2}\right)^{C_{2}(q)} \leq r<1
$$

where

$$
C_{1}(q)=\frac{1}{2(q-2)}, \quad A_{0}=\frac{1}{3.000}, \quad \text { and } \quad C_{2}(q)=2 q+3 .
$$

In the proof of Lemma 3.1 we shall use the magnitude $\alpha(w)$ defined on the $w$-plane punctured at $a_{1}, a_{2}, \ldots, a_{q-1, \infty}$ by

$$
\alpha(w)=\left(1-|z|^{2}\right)\left|F^{\prime}(z)\right|, \quad w=F(z) .
$$

This is a one-valued function of $w$. Next we consider the function

$$
d(w)=\min _{1 \leq \mu \leq q-1}\left|w-a_{\mu}\right|
$$

and show that $\alpha(w)$ satisfies

(i) $\alpha(w) \geq d(w)$,

(ii) $\alpha(w) \leq 2 d(w)\left(|\log d(w)|+\log \frac{1}{\delta}+\Gamma\left(\frac{1}{4}\right)^{4} / 2 \pi^{2}\right)$.

(i) follows from the fact that the Riemann surface contains the disc $\{\xi|| \xi-w \mid<$ $d(w)\}$, since by Schwarz's Lemma applied to the inverse function of

$$
G(s)=F\left(\frac{s+z}{1+\bar{z} s}\right)
$$

in this disc we have

$$
\left|\left(G^{-1}\right)^{\prime}(w)\right|=\left|\frac{1}{G^{\prime}(0)}\right|=\left|\frac{1}{F^{\prime}(z)\left(1-|z|^{2}\right)}\right| \leq \frac{1}{d(w)},
$$

i.e.

$$
\alpha(w)=\left|F^{\prime}(z)\right|\left(1-|z|^{2}\right) \geq d(w) .
$$

(ii) follows from Landau's Theorem applied to

$$
\Phi(s)=\frac{F((s+z) /(1+\bar{z} s))-a_{\mu}}{a_{\nu}-a_{\mu}},
$$

where $a_{\mu}$ is the nearest omitted value and $a_{\nu}$ is any other omitted value.

By Landau's Theorem as in [6],

$$
\left|\Phi^{\prime}(0)\right| \leq 2|\Phi(0)|\left\{|\log | \Phi(0)||+\frac{\Gamma(1 / 4)^{4}}{2 \pi^{2}}\right\} .
$$


On the other hand

$$
\left|\Phi^{\prime}(0)\right|=\frac{\left|F^{\prime}(z)\left(1-|z|^{2}\right)\right|}{\left|a_{\nu}-a_{\mu}\right|}=\frac{\alpha(w)}{\left|a_{\nu}-a_{\mu}\right|}
$$

and

$$
|\Phi(0)|=\frac{\left|F(z)-a_{\mu}\right|}{\left|a_{\nu}-a_{\mu}\right|}=\frac{|d(w)|}{\left|a_{\nu}-a_{\mu}\right|} .
$$

From (3.5), (3.6), and (3.7), we conclude

$$
\begin{aligned}
\alpha(w) & \leq 2|d(w)|\left(|\log | d(w)||+\log \frac{1}{\left|a_{\nu}-a_{\mu}\right|}+\frac{\Gamma(1 / 4)^{4}}{2 \pi^{2}}\right) \\
& \leq 2|d(w)|\left\{|\log | d(w)||+\log \frac{1}{\delta}+\frac{\Gamma(1 / 4)^{4}}{2 \pi^{2}}\right\}
\end{aligned}
$$

i.e. (ii).

Now we define

$$
\begin{aligned}
& E_{\mu}=\left\{w|| w\left|-a_{\mu}\right|<\delta / 2\right\}, \quad \mu=1,2, \ldots, q-1, \\
& E_{q}=\{w|| w \mid>2 / \delta\}, \quad E_{q+1} \text { elsewhere. }
\end{aligned}
$$

Then if $F(z)$ is in $E_{\mu}$, using (i) we obtain

$$
\log \left(\left(1-|z|^{2}\right)\left|F^{\prime}(z)\right|\right) \geq \log \left|F(z)-a_{\mu}\right|,
$$

and since $F(z) \in E_{\mu}$ we have $\left|F(z)-a_{\mu}\right|<\frac{1}{2} \delta<1$, and so

$$
\begin{aligned}
m\left(r, a_{\mu}\right)= & \frac{1}{2 \pi} \int_{E_{\mu}} \log \left|\frac{1}{F\left(r e^{i \theta}\right)-a_{\mu}}\right| d \theta \\
& +\frac{1}{2 \pi} \int_{E_{\mu}^{\prime}} \log ^{+}\left|\frac{1}{F\left(r e^{i \theta}\right)-a_{\mu}}\right| d \theta
\end{aligned}
$$

where $E_{\mu}^{\prime}=\mathrm{C} \backslash E_{\mu}$ is the complementary set of $E_{\mu}$ and where the first integral is taken over the set of values of $\theta$ such that $F\left(r e^{i \theta}\right) \in E_{\mu}$ and similarly the second integral is taken over the set of values of $\theta$ such that $F\left(r e^{i \theta}\right) \in E_{\mu}^{\prime}$.

From (3.10) we deduce

$$
\begin{aligned}
\frac{1}{2 \pi} \int_{E_{\mu}} & \log \left|F\left(r e^{i \theta}\right)-a_{\mu}\right| d \theta \\
& =-m\left(r, a_{\mu}\right)+\frac{1}{2 \pi} \int_{E_{\mu}^{\prime}} \log ^{+}\left|\frac{1}{F\left(r e^{i \theta}\right)-a_{\mu}}\right| d \theta \\
& \geq-m\left(r, a_{\mu}\right), \quad 1 \leq \mu \leq q-1 .
\end{aligned}
$$

Next let us assume $w=F(z) \in E_{q}$; then we have

$$
\left(1-|z|^{2}\right)\left|F^{\prime}(z)\right| \geq \frac{1}{2}|F(z)| \text {. }
$$

In fact, in this case $\left|F(z)-a_{\mu}\right| \geq|F(z)|-\left|a_{\mu}\right|$, and since $\left|a_{\mu}\right| \leq 1 / \delta \leq \frac{1}{2}|F(z)|$, we obtain

$$
\left|F(z)-a_{\mu}\right| \geq|F(z)|-\frac{1}{2}|F(z)|=\frac{1}{2}|F(z)|, \quad \mu=1, \ldots, q-1 .
$$


By (i), we deduce from (3.13)

$$
\left(1-|z|^{2}\right)\left|F^{\prime}(z)\right|=\alpha(w) \geq d(w) \geq \frac{1}{2}|F(z)|,
$$

i.e. (3.12). From (3.12)

$$
\frac{1}{2 \pi} \int_{E_{q}} \log \left(\left(1-r^{2}\right)\left|F^{\prime}\left(r e^{i \theta}\right)\right|\right) d \theta \geq \frac{1}{2 \pi} \int_{E_{q}} \log ^{+}\left|F\left(r e^{i \theta}\right)\right| d \theta-\log 2,
$$

where the integral over $E_{q}$ has the same meaning as above.

On the other hand,

$$
m(r, F)=\frac{1}{2 \pi}\left(\int_{E_{q}}+\int_{E_{q}^{\prime}}\right) \leq \frac{1}{2 \pi} \int_{E_{q}} \log ^{+}\left|F\left(r e^{i \theta}\right)\right| d \theta+\log \frac{2}{\delta},
$$

and so from (3.14) and (3.15)

$$
\frac{1}{2 \pi} \int_{E_{q}} \log \left(\left(1-r^{2}\right)\left|F^{\prime}\left(r e^{i \theta}\right)\right|\right) d \theta \geq m(r, F)-\log \frac{4}{\delta} .
$$

In $E_{q+1}$ we have by (i), $\left(1-|z|^{2}\right)\left|F^{\prime}(z)\right| \geq d(w) \geq \frac{1}{2} \delta$, so that

$$
\frac{1}{2 \pi} \int_{E_{q+1}} \log \left(\left(1-r^{2}\right)\left|F^{\prime}\left(r e^{i \theta}\right)\right|\right) d \theta \geq \log \frac{\delta}{2} .
$$

Now from (3.9), (3.11), (3.16), and (3.17) and using the fact that $\log \left|F^{\prime}(z)\right|$ is harmonic we conclude

$$
\begin{aligned}
\log \left\{\left(1-r^{2}\right)\left|F^{\prime}(0)\right|\right\} & =\frac{1}{2 \pi} \sum_{\mu=1}^{q+1} \int_{E_{\mu}} \log \left\{\left(1-r^{2}\right)\left|F^{\prime}\left(r e^{i \theta}\right)\right|\right\} d \theta \\
& \geq m(r, F)-\sum_{\mu=1}^{q-1} m\left(r, a_{\mu}\right)-\log \left(\frac{8}{\delta^{2}}\right) .
\end{aligned}
$$

Since

$$
m\left(r, a_{\mu}\right) \leq m(r, F)-\log \left|F(0)-a_{\mu}\right|+\log ^{+}\left|a_{\mu}\right|+\log 2
$$

(see Hayman [3, p. 5]), we deduce from (3.2) and (3.18)

$$
\begin{aligned}
-(q-2) m(r, F) \leq & \log \left(1-r^{2}\right)+\log \left|F^{\prime}(0)\right|+\sum_{1}^{q-1} \log ^{+}\left|a_{\nu}\right| \\
& +(q-1) \log 2+\log \left(\frac{8}{\delta^{2}}\right)-(q-2) \log \delta
\end{aligned}
$$

i.e.

$$
\begin{aligned}
(q-2) T(r, F) \geq & \log \frac{1}{1-r^{2}}-\log \left|F^{\prime}(0)\right|-\sum_{1}^{q-1} \log ^{+}\left|a_{\nu}\right| \\
& -(q-1) \log \left(\frac{2}{\delta}\right)-\log \left(\frac{8}{\delta^{2}}\right) .
\end{aligned}
$$

We also have, by hypotheses for $\mu=1,2, \ldots, q-1$,

$$
\delta \leq\left|F(0)-a_{\mu}\right| \leq|F(0)|+\left|a_{\mu}\right| \leq \frac{2}{\delta},
$$


so that

$$
\delta \leq d(F(0)) \leq \frac{2}{\delta}
$$

Thus we obtain by (ii)

$$
\begin{aligned}
\left|F^{\prime}(0)\right| & \leq \frac{4}{\delta}\left(\log \frac{2}{\delta}+\log \frac{1}{\delta}+\frac{\Gamma(1 / 4)^{4}}{2 \pi^{2}}\right) \\
& \leq \frac{8}{\delta}\left(\log \frac{1}{\delta}+3\right)
\end{aligned}
$$

i.e.

$$
\begin{aligned}
\log \left|F^{\prime}(0)\right| & \leq \log \frac{1}{\delta}+\log 8+\log ^{+} \log \frac{1}{\delta}+\log 3+\log 2 \\
& <2 \log \frac{1}{\delta}+\log 48
\end{aligned}
$$

We also have

$$
\sum_{1}^{q-1} \log ^{+}\left|a_{\mu}\right| \leq(q-1) \log \frac{1}{\delta} .
$$

From (3.19), (3.20), and (3.21), we conclude

$$
\begin{aligned}
(q-2) T(r, F) \geq & \log \frac{1}{1-r^{2}}-2 \log \frac{1}{\delta}-\log 48-(q-1) \log \frac{1}{\delta} \\
& -(q-1) \log \frac{2}{\delta}-\log \left(\frac{8}{\delta^{2}}\right) \\
\geq & \log \frac{1}{1-r}-(2 q+2) \log \frac{1}{\delta}-(q+3) \log 2-\log 48
\end{aligned}
$$

hence

$$
(q-2) T(r, F) \geq \frac{1}{2} \log \frac{1}{1-r}
$$

if

$$
\frac{1}{2} \log \frac{1}{1-r}>\log \left\{48 \cdot 2^{(q+3)} \cdot \delta^{-(2 q+2)}\right\}
$$

i.e. if

$$
r>1-\left\{\frac{1}{48} \cdot 2^{-(q+3)} \cdot \delta^{2 q+2}\right\}^{2}
$$

Thus we get

$$
T(r, F) \geq \frac{1}{2(q-2)} \log \frac{1}{1-r}
$$

if

$$
r>\overline{\mathrm{i}}-\frac{1}{3000}\left(\frac{1}{2} \delta^{2}\right)^{2 q+3}
$$

which is (3.3). 
3.2 .

LEMMA 3.2. Let $p$ be a positive integer and $x$ a positive number. Then there exist $a_{1}$ such that ex $<\left|a_{1}\right|<10$ ex and a function

$$
F(z)=a_{1} z+a_{p+1} z^{p+1}+\cdots
$$

regular in $|z|<1$, univalent in $z<\sqrt{2}-1$, and assuming the values $i, 0$ no more than once in $|z|<1$.

Furthermore for $x \geq 2$ the function $F$ satisfies

$$
T(r, F) \geq \frac{1}{192 p} \log \frac{1}{1-r}, \quad \mathbf{R}_{p} \leq r<1,
$$

where

$$
\mathbf{R}_{p}=1-\frac{A_{0}}{p}(e x)^{-26 p}
$$

and $A_{0}$ is a positive constant.

Let $E_{1}$ be the set $\{0, \pm e x, i, \infty\}$ and let $\mathbf{R}$ be the universal covering surface over the complement of $E_{1}$. Let $\mathbf{R}_{1}, \mathbf{R}_{2}$ be the surfaces obtained by cutting $\mathbf{R}$ from $e x$ to $+\infty$ along the real axis and $\mathbf{R}_{3}, \mathbf{R}_{4}$ those obtained by cutting $\mathbf{R}$ from $-e x$ to $-\infty$. Let $\mathbf{R}_{5}$ be the plane cut from $e x$ to $+\infty$ and from $-e x$ to $-\infty$ along the real axis and finally let $\mathbf{R}_{0}$ be obtained by joining $\mathbf{R}_{1}, \mathbf{R}_{2}$ to $\mathbf{R}_{5}$ on the segment $(e x, \infty)$ and $\mathbf{R}_{3}, \mathbf{R}_{\mathbf{4}}$ to $\mathbf{R}_{5}$ along the segment $(-\infty,-e x)$.

The Riemann surface $\mathbf{R}_{0}$ obtained in this way contains none of the points $\pm e x, \infty$ in any sheet and contains the points 0 and $i$ exactly once, those in the sheet $\mathbf{R}_{5}$. $\mathbf{R}_{0}$ is simply connected and since it does not contain points over $\pm e x, \infty, \mathbf{R}_{0}$ is hyperbolic.

Therefore there is a conformal map $F_{0}$ from the unit disc $\{z|| z \mid<1\}$ onto $\mathbf{R}_{0}$,

$$
F_{0}(z)=b_{1} z+b_{2} z^{2}+\cdots, \quad b_{1}>0 .
$$

By the construction of $\mathbf{R}_{0}$ and $F_{0}$ we deduce that $F_{0}$ assumes $i, 0$ precisely once and that it never assumes $\pm e x, \infty$. This implies that $F_{0}$ is subordinate to the function $G$ which maps $\{z|| z \mid<1\}$ onto the universal covering over the plane punctured at $\pm e x, \infty$ satisfying

$$
G(0)=0, \quad G^{\prime}(0)>0 .
$$

This function $G$ maps the sheet $\mathbf{R}_{5}$ onto a quadrilateral $Q$ in the unit disc, bounded by four quarter circles, orthogonal to the circumference $\{z|| z \mid=1\}$, joining the points $z=1, i,-1,-i$ in the form $(1, i),(i,-1),(-1,-i)$, and $(-i, 1)$.

$Q$ contains the disc $\{z|| z \mid<\sqrt{2}-1\}$ and since $F_{0}(z)$ is subordinate to $G(z), F_{0}(z)$ maps this disc onto a subset of the sheet $\mathbf{R}_{5}$; therefore $F_{0}(z)$ is univalent in $\{z|| z \mid<\sqrt{2}-1\}$.

By Koebe's Theorem we have

$$
e x>\frac{b_{1}(\sqrt{2}-1)}{4},
$$

and since the inverse function $z=\Phi(w)$ maps the disc $\{w|| w \mid<e x\}$ into the disc $\{z|| z \mid<1\}$, we can apply Schwarz's Lemma and obtain

$$
b_{1}^{-1}=\Phi^{\prime}(0)<(e x)^{-1} \text {. }
$$


Hence we have

$$
e x<b_{1}<\frac{4}{\sqrt{2}-1} e x<10 e x .
$$

Thus we have constructed a function (3.22) in the case $p=1$. When $p>1$ we proceed as follows.

If $p$ is odd so that $i^{p} \neq \pm 1$, we write $E_{p}=\left\{ \pm(e x)^{p}, 0, i^{p}, \infty\right\}$ and define $F_{p}(z)$ as above with $E_{p}$ instead of $E_{1}$ and

$$
F(z)=\left\{F_{p}\left(z^{p}\right)\right\}^{1 / p} .
$$

A standard argument in the elementary theory of univalent functions shows that $F(z)$ has the required properties, i.e. it has an expansion (3.22) where $e x<\left|a_{1}\right|<$ $10 e x$ is regular in $|z|<1$, univalent in $|z|<\sqrt{2}-1$ and assumes the values $i, 0$ no more than once in $|z|<1$.

If $p$ is even we consider the set $E_{p}^{\prime}=\left\{ \pm(e x)^{p}, 0,-i^{p+1}, \infty\right\}$ instead of $E_{p}$ and the function $i F_{p}(-i z)$ instead of $F_{p}(z)$ and then define $F(z)$ by $(3.25)$ again.

Finally we prove (3.23). We shall make use of Lemma 3.1. tions.

We consider again the case $p$ odd. The case $p$ even follows with small modifica-

According to Lemma 3.1, any function $F_{5}$ mapping conformally the urit disc onto the universal covering surface over the Riemann sphere punctured at the points of $E_{p}=\left\{0, \pm(e x)^{p}, i^{p}, \infty\right\}$, such that

$$
\left|F_{5}(0)-a\right| \geq \delta \quad \text { for every } a \in E_{p}, \quad\left|F_{5}(0)\right| \leq \frac{1}{\delta}
$$

where $\delta \leq \min \left\{1,(e x)^{p},(e x)^{p}-1\right\}$ and $1 / \delta \geq\left\{(e x)^{p}, 1\right\}$, i.e. such that (3.1) and (3.2) are satisfied, has a characteristic function $T\left(r, F_{5}\right)$ such that

$$
T\left(r, F_{5}\right) \geq \frac{1}{6} \log \frac{1}{1-r}, \quad 1-A_{0}\left(\frac{1}{2} \delta^{2}\right)^{13} \leq r<1,
$$

where $A_{0}$ is an absolute constant.

For $x \geq 2$, we can take $\delta=1 /(e x)^{p}$.

First we shall consider a translate $F_{p a}$ of $F_{p}$ where

$$
F_{p a}(z)=F_{p}\left(\frac{z-a}{1-\bar{a} z}\right)
$$

for some $a$ such that $|a|<1$.

We choose the branch of $F_{5}$ at $z=0$, such that $F_{5}(0)=1 /(e x)^{p}$, so that for $x \geq 2(3.26)$ is satisfied and then consider a translate $F_{p a}$ of $F_{p}$ such that $F_{p a}(0)=1 /(e x)^{p}$. It is clear that we can define the function

$$
\omega(z)=F_{p a}^{-1} \circ F_{5}
$$

in a neighborhood of $z=0$, so that $\omega^{\prime}(0)=0$, and by the construction of $F_{p a}$ and $F_{5}, \omega$ can be continued without limit in the disc and since this is a simply connected set, we can make use of the monodromy theorem to obtain a function from the whole unit disc onto itself such that $\omega(0)=0$.

From (3.28) we obtain

$$
F_{5}(z)=F_{p a}(\omega(z))
$$


i.e. we conclude that $F_{5}$ is subordinate to $F_{p a}$. Hence $F_{p a}$ also satisfies (3.27):

$$
T\left(r, F_{p a}\right) \geq \frac{1}{6} \log \frac{1}{1-r}, \quad 1-A_{0}\left(\frac{1}{2}(e x)^{-2 p}\right)^{13} \leq r<1 .
$$

Next, we shall prove a similar condition for $F_{p}$. To do this, we shall use the Ahlfors-Shimizu characteristic $T_{0}$ instead of the Nevanlinna characteristic $T . T_{0}$ and $T$ differ only by a bounded term so that we obtain equivalent statements.

We recall that for a meromorphic function $G, T_{0}$ is defined by

$$
T_{0}(r, G)=\int_{0}^{r} \frac{A(t)}{t} d t
$$

where $A(t)$ is the area, with due regard to multiplicity, of the image on the Riemann sphere of $\{z|| z \mid \leq t\}$ by $G$.

If $G$ is regular then

$$
T_{0}(r, G)=\frac{1}{2 \pi} \int_{0}^{2 \pi} \log \sqrt{1+\left|G\left(r e^{i \theta}\right)\right|^{2}} d \theta-\log \sqrt{1+G(0)^{2}} .
$$

Therefore, using the inequality

$$
\log ^{+} x \leq \log \sqrt{1+x^{2}} \leq \log ^{+} x+\frac{1}{2} \log 2,
$$

and the fact that for $G$ regular

$$
T(r, G)=\frac{1}{2 \pi} \int_{0}^{2 \pi} \log ^{+}\left|G\left(r e^{i \theta}\right)\right| d \theta,
$$

we obtain

$$
T(r, G)-\log \sqrt{1+G(0)^{2}} \leq T_{0}(r, G) \leq T(r, G)-\log \sqrt{1+G(0)^{2}}+\frac{1}{2} \log 2 .
$$

First we show that

$$
\left|\frac{z+a}{1+\bar{a} z}\right| \geq 1-\frac{2}{1-|a|}(1-r), \quad|z|=r .
$$

In fact, we have

$$
\left|\frac{z+a}{1+\bar{a} z}\right| \geq \frac{r-a}{1-|\bar{a}| r}
$$

whence

$$
1-\left|\frac{z+a}{1+\bar{a} z}\right| \leq 1-\frac{r-|a|}{1-|a| r}=\frac{(1-r)(1+|a|)}{1-|a| r} \leq \frac{2}{1-|a|}(1-r),
$$

which yields (3.31).

The function $w=(z+a) /(1+\bar{a} z)$ maps the disc $\{z|| z \mid<t\}, t>|a|$, conformally onto a disc containing the origin, establishing also a one-to-one and continuous correspondence between the circumference $\{z|| z \mid=t\}$ and the boundary of that disc.

On the other hand by $(3.31)$

$$
|w(z)| \geq 1-\frac{2}{1-|a|}(1-t), \quad|z|=t
$$


i.e. we can conclude that the disc contains the disc with center at the origin

$$
\left\{w|| w \mid \leq 1-\frac{2}{1-|a|}(1-t)\right\} \text {. }
$$

Now since $F_{p}(z)=F_{p a}((z+a) /(1+\bar{a} z))$, we deduce from the above considerations that for every solution in

$$
\left\{w|| w \mid \leq 1-\frac{2}{1-|a|}(1-t)\right\}
$$

of $F_{p a}(w)=\varsigma$, there is at least one solution of $F_{p}(z)=\varsigma$ in $\{z|| z \mid \leq t\}$ and different solutions of the first equation give rise to different solutions of the last equation.

We now recall that (see Hayman [3, p. 13]),

$$
A_{F_{p a}}(t)=\int_{S} n_{F_{p a}}(t, \zeta) d \mu(\varsigma), \quad A_{F_{p}}(t)=\int_{S} n_{F_{p}}(t, \zeta) d \mu(\zeta),
$$

where $S$ is the Riemann sphere, $d \mu$ is the normalized area element in $S$, and $n_{F_{p a}}(t)$ and $n_{F_{p}}(t)$ are the number of roots of $F_{p a}(w)=\varsigma$ and $F_{p}(z)=\varsigma$ in $|w| \leq t,|z| \leq t$, respectively.

Therefore we conclude from what we have said above

$$
A_{F_{p}}(t) \geq A_{F_{p a}}\left(1-\frac{2}{1-|a|}(1-t)\right), \quad t>|a| .
$$

On the other hand $F_{p}$ is univalent in $\{z|| z \mid<\sqrt{2-1}\}$ and $F_{p}(0)=0$. Thus by Koebe's Theorem the disc $\left\{w|| w \mid<b_{1}(\sqrt{2}-1) / 4\right\}$, is covered by the image of $\{z|| z \mid<\sqrt{2}-1\}$ by $F_{p}$.

Therefore, since $F_{p}(-a)=F_{p a}(0)=(e x)^{-p}$, we can find $a$ in $\{z|| z \mid<\sqrt{2}-1\}$ provided that

$$
\frac{b_{1}(\sqrt{2}-1)}{4}>\frac{1}{(e x)^{p}} .
$$

But, by (3.24), $\left|b_{1}\right|>(e x)>10(e x)^{-p}$, since $x \geq 2$, so that the above condition is satisfied.

Now, by an inequality for univalent functions (see Hayman $[2$, p. 4]), we obtain

$$
\left|F_{p}(-a)\right| \geq \frac{\left|b_{1}\right||a|}{(1+|a| /(\sqrt{2}-1))^{2}} \geq \frac{\left|b_{1}\right||a|}{4}
$$

and since $\left|b_{1}\right| \geq(e x)^{p}$, we also have

$$
|a| \leq \frac{4\left|F_{p}(-a)\right|}{\left|b_{1}\right|}=\frac{4}{\left|b_{1}\right|(e x)^{p}} \leq \frac{4}{(e x)^{2 p}} \leq \frac{1}{e^{2}} \leq \frac{1}{7},
$$

since $x \geq 2$.

Next we show that

$$
1-\frac{2}{1-|a|}(1-t) \geq t^{4}, \quad \frac{2}{3} \leq t \leq 1 .
$$


In fact if $|a|<1 / 7$, and $2 / 3 \leq t \leq 1$,

$$
\begin{aligned}
1-t^{4}-\frac{2}{1-|a|}(1-t) & \geq 1-t^{4}-\frac{7}{3}(1-t) \\
& =(1-t)\left\{1+t+t^{2}+t^{3}-\frac{7}{3}\right\} \\
& \geq(1-t)\left\{1+\frac{2}{3}+\frac{4}{9}+\frac{8}{27}-\frac{7}{3}\right\}=\frac{2}{27}(1-t)>0 .
\end{aligned}
$$

From (3.30), (3.32), and (3.33) and since $F_{p}(0)=0$ we have

$$
\begin{aligned}
T\left(r, F_{p}\right)+\frac{1}{2} \log 2 & \geq T_{0}\left(r, F_{p}\right)=\int_{0}^{r} A_{F_{p}}(t) \frac{d t}{t} \\
& \geq \int_{2 / 3}^{r} A_{F_{p a}}\left\{1-\frac{2}{1-|a|}(1-t)\right\} \frac{d t}{t} \geq \int_{2 / 3}^{r} A_{F_{p a}}\left(t^{4}\right) \frac{d t}{t} \\
& =\int_{(2 / 3)^{4}}^{r^{4}} A_{F_{p a}}(s) \frac{d s}{s} \geq \frac{1}{4} \int_{1 / 5}^{r^{4}} A_{F_{p a}}(s) \frac{d s}{s} \\
& =\frac{1}{4}\left\{T_{0}\left(r^{4}, F_{p a}\right)-T_{0}\left(\frac{1}{5}, F_{p a}\right)\right\} \\
& >\frac{1}{4}\left\{T\left(r^{4}, F_{p a}\right)-T\left(\frac{1}{5}, F_{p a}\right)-\frac{1}{2} \log 2\right\} .
\end{aligned}
$$

Now we obtain a lower bound for $T\left(r^{4}, F_{p a}\right)$ and an upper bound for $T\left(1 / 5, F_{p a}\right)$. To do the first point, we make use of (3.29) and obtain

$$
\begin{aligned}
T\left(r^{4}, F_{p a}\right) & \geq \frac{1}{6} \log \frac{1}{1-r^{4}} \geq \frac{1}{6} \log \frac{1}{4(1-r)} \\
& =\frac{1}{6}\left(\log \frac{1}{1-r}-\log 4\right) \geq \frac{1}{12} \log \frac{1}{1-r}
\end{aligned}
$$

if

$$
r^{4} \geq 1-A_{0}\left(\frac{1}{2}(e x)^{-2 p}\right)^{13}
$$

and if

$$
\log \frac{1}{1-r} \geq 2 \log 4, \quad \text { i.e. } r \geq 1-e^{-16},
$$

both conditions can be included in (3.36) after decreasing $A_{0}$ if necessary.

Next we obtain an upper bound for $T\left(1 / 5, F_{p a}\right)$.

$F_{p}$ is univalent in $\{z|| z \mid<\sqrt{2}-1\}$, therefore by an inequality for univalent functions (see Hayman [2, p. 4]), we get

$$
\left|F_{p}(z)\right|<\frac{\left|b_{1}\right| r_{0}^{2}|z|}{\left(r_{0}-|z|\right)^{2}}, \quad|z|<r_{0}=\sqrt{2}-1 .
$$

On the other hand for $|\xi| \leq 1 / 5,|a| \leq 1 / 7$, we have

$$
\left|\frac{\xi-a}{1-\bar{a} z}\right| \leq \frac{|\xi|+|a|}{1+|a||\xi|} \leq \frac{1 / 5+1 / 7}{1+1 / 35}=\frac{1}{3},
$$


so that for $|\xi| \leq 1 / 5$

$$
F_{p a}(\xi)=F_{p}\left(\frac{\xi-a}{1-\bar{a} \xi}\right)=F_{p}(z)
$$

where $|z| \leq 1 / 3$. Hence since $r_{0}=\sqrt{2}-1>2 / 5$, we conclude from (3.37)

$$
\left|F_{p}(z)\right| \leq \frac{\left|b_{1}\right||z|}{\left(1-|z| / r_{0}\right)^{2}} \leq \frac{1 / 3\left|b_{1}\right|}{(1-5 / 6)^{2}}=12\left|b_{1}\right| \leq 120(e x)^{p} .
$$

From (3.38), we obtain

$$
T\left(1 / 5, F_{p a}\right) \leq \log \left\{120(e x)^{p}\right\} .
$$

Therefore if (3.36) holds, we have by (3.34), (3.35), and (3.39)

$$
\begin{aligned}
T\left(r, F_{p}\right) & \geq \frac{1}{4}\left\{\frac{1}{12} \log \frac{1}{1-r}-\log \left\{120(e x)^{p}\right\}-\frac{1}{2} \log 2\right\}-\frac{1}{2} \log 2 \\
& \geq \frac{1}{96} \log \frac{1}{1-r}
\end{aligned}
$$

if

and this will happen if

$$
\frac{1}{24} \log \frac{1}{1-r} \geq \log \left\{120(e x)^{p}\right\}+\frac{3}{2} \log 2,
$$

$$
1>r \geq 1-\left(480(e x)^{p}\right)^{-24} .
$$

Again we can put together (3.36) and (3.41) in

$$
1>r^{4} \geq 1-A_{0}(e x)^{-26 p},
$$

with a new $A_{0}$.

Now, from (3.25) and (3.40), we deduce

$$
T(r, F)=\frac{1}{p} T\left(r^{p}, F_{p}\right) \geq \frac{1}{96 p} \log \frac{1}{1-r^{p}}
$$

if

$$
1>r^{4 p} \geq 1-A_{0}(e x)^{-26 p}
$$

whence

$$
\begin{aligned}
T(r, F) & \geq \frac{1}{96 p} \log \frac{1}{p(1-r)}=\frac{1}{96 p}\left(\log \frac{1}{1-r}-\log p\right) \\
& \geq \frac{1}{192 p} \log \frac{1}{1-r}
\end{aligned}
$$

if, in addition to (3.44), we have

$$
\log \frac{1}{1-r} \geq 2 \log p, \quad \text { i.e. } r \geq 1-\frac{1}{p^{2}} .
$$

Using the fact that $4 p(1-r) \geq 1-r^{4 p}$, we conclude that (3.44) and (3.46) will be satisfied if

$$
1>r \geq 1-\frac{A_{0}}{p}(e x)^{-26 p},
$$

with $A_{0}$ an absolute constant.

We have that (3.45) holds in the range (3.47), so that we have proved (3.23). This completes the proof of Lemma 3.2. 


\section{3 .}

LEMMA 3.3. Suppose that $a_{1}, \ldots, a_{p}$ are preassigned complex numbers, not all zero, and write $M=\sum_{\nu=1}^{p}\left|a_{\nu}\right|$ and $\mu>e M$.

Then the function

$$
\omega(z)=\frac{\sum_{\nu=1}^{p} a_{\nu} z^{\nu}+\mu z^{2 p}}{\mu+\sum_{\nu=1}^{p} \bar{a}_{\nu} z^{2 p-\nu}}
$$

has precisely $2 p$ zeros and no poles in the disc

$$
\{z|| z \mid \leq \exp (-1 / 2 p)\} \text {. }
$$

Furthermore the function $\omega$ satisfies the following inequality:

$$
1-16 p^{2}(1-|z|)<|\omega(z)|<1-\frac{1}{8 p}(1-|z|) .
$$

First we observe that $|\omega(z)|=1$ for $|z|=1$, $\omega$ has no poles in $\{z|| z \mid<1\}$, and by Rouché's Theorem $\omega$ has $2 p$ zeros in the unit disc. Next we show that these $2 p$ zeros are in the smaller disc (3.49).

We write $r_{p}=\exp \{-1 / 2 p\}$. Then for $r_{p}<|z|<1$, we have

$$
\left|\sum_{\nu=1}^{p} a_{\nu} z^{\nu}\right| \leq M<\frac{\mu}{e}=\mu r_{p}^{2 p}<\left|\mu z^{2 p}\right|,
$$

i.e. $\omega(z) \neq 0$ for $r_{p}<|z|<1$, and therefore if $z_{\nu}, 1 \leq \nu \leq 2 p$, are the zeros of $\omega$ in the unit disc, we have

$$
\left|z_{\nu}\right| \leq r_{p}, \quad 1 \leq \nu \leq 2 p .
$$

We now consider the function

$$
\Pi(z)=\prod_{\nu=1}^{2 p} \frac{z-z_{\nu}}{1-\bar{z}_{\nu} z} .
$$

Then $\Phi(z)=\omega(z) / \Pi(z)$ is regular and not zero in $\{z|| z \mid \leq 1\}$ and $|\Phi(z)|=1$ on $|z|=1$. Thus $|\Phi(z)| \equiv 1$ and

$$
\omega(z)=e^{i \theta_{0}} \Pi(z)=e^{i \theta_{0}} \prod_{\nu=1}^{2 p} \frac{z-z_{\nu}}{1-\bar{z}_{\nu} z},
$$

where $\theta_{0}$ is a constant such that $0 \leq \theta_{0} \leq 2 \pi$.

Using (3.49) we deduce that for $|z|=r>r_{p}$, we have

$$
\left(\frac{r-r_{p}}{1-r_{p} r}\right)^{2 p} \leq|\Pi(z)|=|\omega(z)| \leq\left(\frac{r+r_{p}}{1+r_{p} r}\right)^{2 p} .
$$

We write

$$
x=\frac{r-r_{p}}{1-r_{p} r}, \quad y=\frac{r_{p}+r}{1+r_{p} r},
$$

and obtain

$$
\begin{aligned}
1-x^{2 p} & =(1-x)\left(1+x+\cdots+x^{2 p-1}\right)<2 p(1-x) \\
& =2 p \frac{(1-r)\left(1+r_{p}\right)}{1-r_{p} r}<\frac{4 p}{1-r_{p}}(1-r) .
\end{aligned}
$$


On the other hand if $t=1 / 2 p$, so that $0<t \leq 1 / 2$, we have $1-e^{-t}=t e^{-\tau}, 0<$ $\tau<1 / 2$, hence

$$
1-e^{-t}>t e^{-1 / 2}>\frac{1}{2} t, \quad r_{p}=e^{-t}<1-\frac{1}{2} t=1-\frac{1}{4 p} .
$$

Thus we obtain from (3.53)

$$
1-x^{2 p}<\frac{4 p}{1-r_{p}}(1-r)<16 p^{2}(1-r)
$$

Now, (3.52) and (3.54) yield the left-hand inequality of (3.50). To prove the right-hand inequality we obtain an upper bound for $y^{2 p}$. We have

$$
1-y^{2 p} \geq 1-y=\frac{\left(1-r_{p}\right)(1-r)}{1+r_{p} r} \geq \frac{1}{2}\left(1-r_{p}\right)(1-r)>\frac{1-r}{8 p} .
$$

From this follows the right-hand inequality of (3.50).

3.4 .

LEMMA 3.4. Suppose given the complex numbers $a_{1}, a_{2}, \ldots, a_{p}$, not all zero, and write $M=\sum_{\nu=1}^{p}\left|a_{\nu}\right|$.

Then there exists $F_{p}(z)$ regular in $|z|<1$, assuming $i$ and 0 no more than $2 p$ times, and with a power series development

$$
F_{p}(z)=a_{1} z+a_{2}^{2}+\cdots+a_{p} z^{p}+O\left(z^{p+1}\right),
$$

near $z=0$. Furthermore

$$
\left|F_{p}(z)\right|<40 e M \quad \text { in }|z|<\frac{\sqrt{2}-1}{2}
$$

and if $M \geq 2$, we have

$$
T\left(r, F_{p}\right) \geq \frac{1}{8000 p^{3}} \log \frac{1}{1-r}, \quad R_{p}^{\prime} \leq r<1,
$$

where

$$
R_{p}^{\prime}=1-\frac{A_{0}}{p^{3}}(16 e M)^{-1000 p^{4}}
$$

Let $F(z)$ be the function whose existence is asserted in Lemma 3.2 with $x=M$. Let us write $a_{1}=\mu$, then $e M<\mu<10 e M$, and consider the function

$$
\omega(z)=\frac{\sum_{\nu=1}^{p} a_{\nu} z^{\nu}+\mu z^{2 p}}{\mu+\sum_{\nu=1}^{p} \bar{a}_{\nu} z^{2 p-\nu}}
$$

as in Lemma 3.3.

Then we define

$$
F_{p}(z)=F(\omega(z))
$$

One can check that

$$
\omega(z)=\mu^{-1} \sum_{\nu=1}^{p} a_{\nu} z^{\nu}+O\left(z^{p+1}\right)
$$

and so

$$
F_{p}(z)=\mu \omega(z)+O\left(z^{p+1}\right)
$$

whence we conclude (3.55). 
The equation $\omega(z)=\varsigma$ has precisely $2 p$ roots in $\{z|| z \mid<1\}$ for any $\zeta$ in $\{\varsigma|| \zeta \mid<$ $1\}$, and since the equations $F(\omega)=0$ and $F(\omega)=i$ have at most one root in the unit disc, we deduce that the equations $F_{p}(z)=0$ and $F_{p}(z)=i$ have at most $2 p$ roots in $\{z|| z \mid<1\}$.

Since $F(z)$ is univalent in $\left\{z|| z \mid<r_{0}=\sqrt{2}-1\right\}$, we have by a classical inequality for univalent functions

$$
|F(z)|<\frac{\mu r_{0}^{2}|z|}{\left(r_{0}-|z|\right)^{2}}, \quad|z|<r_{0} .
$$

By Schwarz's Lemma, $|\omega(z)| \leq|z|$ for $|z|<1$ and so for $|z|=r_{0} / 2$ we obtain

$$
\left|F_{p}(z)\right| \leq \frac{\mu r_{0}^{2}|\omega(z)|}{\left(r_{0}-|\omega(z)|\right)^{2}} \leq 4 \mu|\omega(z)| \leq 40 e M|z|<40 e M,
$$

which is (3.56).

To prove (3.57), we shall make use of (3.23) and the definition of $F_{p}$ in (3.58). We shall also use the Ahlfors-Shimizu characteristic $T_{0}$ instead of the Nevanlinna characteristic $T$ as we did in the proof of Lemma 3.2.

First we show that

$$
A_{F_{p}}(t) \geq A_{F}\left(1-16 p^{2}(1-t)\right), \quad t \geq r_{p}=\exp \{-1 / 2 p\} .
$$

This follows from the definition of $F_{p}$ in (3.58) and the fact that the number of roots in $\{z|| z \mid \leq t\}$ of any equation of the form

$$
F_{p}(z)=\varsigma
$$

is not less than the number of the roots in $\left\{\omega|| \omega \mid \leq 1-16 p^{2}(1-t)\right\}$ of the corresponding equation

$$
F(\omega)=\varsigma .
$$

To see this, suppose that $\omega_{0}$ is a root of $(3.61)$ in $\left\{\omega|| \omega \mid \leq 1-16 p^{2}(1-t)\right\}$. Then we prove that the equation

$$
\omega(z)=\omega_{0}
$$

must have at least one solution in $\{z|| z \mid \leq t\}$, say $z_{0}$.

In fact, the image by $\omega$ of $\{z|| z \mid<t\}$ is a domain $D(t)$ contained in $\{\omega|| \omega<$ $1\}$ containing the origin, since $\omega(z)$ has $2 p$ zeros in the smaller disc $\{z|| z \mid \leq$ $\exp (-1 / 2 p)\}$, and whose boundary is contained in the image of $\{z|| z \mid=t\}$. Then using the left-hand inequality of $(3.50)$ we have

$$
|\omega(z)|>1-16 p^{2}(1-t), \quad|z|=t, t>\exp \{-1 / 2 p\}
$$

so that we can conclude that $D(t)$ contains the disc $\left\{\omega|| \omega \mid<1-16 p^{2}(1-t)\right\}$, i.e. the equation (3.62) admits at least one solution $z_{0}$ in $\{z|| z \mid \leq t\}$.

Now

$$
F_{p}\left(z_{0}\right)=F\left(\omega\left(z_{0}\right)\right)=F\left(\omega_{0}\right)=\varsigma,
$$

i.e. $z_{0}$ is a solution of (3.60).

Therefore for every solution of $(3.61)$ in $\left\{\omega|| \omega \mid \leq 1-16 p^{2}(1-t)\right\}$ there is at least one solution of (3.60) in $\{z|| z \mid \leq t\}$ and different solutions of (3.61) yield, in this way, different solutions of $(3.60)$. 
As in Lemma 3.2 we recall that (Hayman [3, p. 13])

$$
A_{F_{p}}(t)=\int_{S} n_{F_{p}}(t, \zeta) d \mu(\varsigma), \quad A_{F}(t)=\int_{S} n_{F}(t, \zeta) 0 d \mu(\zeta),
$$

where $S$ is the Riemann sphere, $d \mu$ is the normalized area element in $S$, and $n_{F_{p}}(t)$, $n_{F}(t)$ are the number of roots of (3.60) and (3.61) respectively, in the disc of radius $t$.

From all these considerations we conclude (3.59).

To prove (3.57), we shall make use of the following inequality:

$$
3 n(1-t)>1-t^{3 n}>n(1-t), \quad \frac{1}{5} \leq t^{3 n}<1, n \geq 1
$$

In fact, let us observe that the quotient

$$
\frac{1-t^{3 n}}{1-t}=1+t+t^{2}+\cdots+t^{3 n-1}
$$

increases with $t$, so that it is enough to consider the case $t^{3 n}=1 / 5$ for the righthand inequality in (3.64). The left-hand inequality is obvious.

We write $h=1 / n, a=\frac{1}{3} \log 5$, and note that

$$
\frac{1-t}{1-t^{3 n}}=\frac{5}{4}\left(1-e^{-a h}\right)<\frac{5 a h}{4}=\frac{5 \log 5}{12 n}<\frac{1}{n},
$$

which proves (3.64).

Now we make use of (3.59) and apply the right-hand inequality of (3.64) with $n=16 p^{2}$. Let $r^{\prime}=5^{-1 / 3 n}$ and observe that $r^{\prime} \geq r_{p}$. Then for $r>r^{\prime}$ we obtain

$$
\begin{aligned}
T_{0}\left(r, F_{p}\right)-T_{0}\left(r^{\prime}, F_{p}\right) & =\int_{r^{\prime}}^{r} A_{F_{p}}(t) \frac{d t}{t} \geq \int_{r^{\prime}}^{r} A_{F}\left\{(1-n(1-t)\} \frac{d t}{t}\right. \\
& \geq \int_{r^{\prime}}^{r} A_{F}\left(t^{3 n}\right) \frac{d t}{t}=\frac{1}{3 n} \int_{r^{\prime 3 n}}^{r^{3 n}} A_{F}(s) \frac{d s}{s} \\
& =\frac{1}{3 n}\left\{T_{0}\left(r^{3 n}, F\right)-T_{0}\left(\frac{1}{5}, F\right)\right\} .
\end{aligned}
$$

Next we find an upper bound for $T_{0}(1 / 5, F)$ and a lower bound for $T_{0}\left(r^{3 n}, F\right)$.

To obtain an upper bound for $T_{0}(1 / 5, F)$, we observe that $F(z)$ is univalent in $|z|<r_{0}$, where $r_{0}=\sqrt{2}-1>2 / 5$. Thus for $|z|=1 / 5<(1 / 2) r_{0}$, we have by an inequality for univalent functions (Hayman [2, p. 4]),

$$
|F(z)| \leq \frac{\left|F^{\prime}(0)\right||z| r_{0}^{2}}{\left(r_{0}-|z|\right)^{2}} \leq 4|z|\left|F^{\prime}(0)\right| \leq \frac{4}{5} \mu<8 e M .
$$

Hence

$$
T_{0}\left(\frac{1}{5}, F\right) \leq T\left(\frac{1}{5}, F\right)+\frac{1}{2} \log 2 \leq \log (8 e M)+\frac{1}{2} \log 2 .
$$

By (3.23) in Lemma 3.2

$$
T\left(r^{3 n}, F\right) \geq \frac{1}{192 p} \log \frac{1}{1-r^{3 n}} \geq \frac{1}{192 p}\left\{\log \frac{1}{1-r}-\log 3 n\right\}
$$

if

$$
r^{3 n} \geq 1-\frac{A_{0}}{p}(e M)^{-26 p}
$$


Thus by (3.65), (3.66), and (3.67) we have

$$
\begin{aligned}
T\left(r, F_{p}\right) & \geq T_{0}\left(r, F_{p}\right)-\frac{1}{2} \log 2 \\
& \geq \frac{1}{3 n}\left\{\frac{1}{192 p}\left\{\log \frac{1}{1-r}-\log 3 n\right\}-\log (8 e M)-\frac{1}{2} \log 2\right\}-\frac{1}{2} \log 2
\end{aligned}
$$

in the range (3.68).

From (3.69) we obtain

$$
T\left(r, F_{p}\right) \geq \frac{1}{8000 p^{3}} \log \frac{1}{1-r}
$$

if (3.68) holds, and if in addition

$$
r \geq 1-A(16 e M)^{-1000 p^{4}} .
$$

We make use again of the inequality

$$
1-r^{3 n} \leq 3 n(1-r)=48 p^{2}(1-r)
$$

and conclude that (3.68) will be satisfied if

$$
r \geq 1-\frac{A_{0}}{p^{3}}(e M)^{-26 p}
$$

(3.71) and (3.72) will be satisfied simultaneously if

$$
r \geq 1-\frac{A_{0}}{p^{3}}(16 e M)^{-1000 p^{4}},
$$

after decreasing $A_{0}$ if necessary.

But (3.70) and (3.73) yield (3.57) and the proof of Lemma 3.4 is complete.

4. Proof of Theorem 1. We prove Theorem 1 by showing that given an arbitrary function $\Phi(r)$ satisfying (2.1), there is an integral function $f$ such that

$$
N(r, 0, f)+N(r, i, f)+N(r, \infty, f)<\frac{1}{2} T(r, f),
$$

for

$$
\rho_{k}-\rho_{k}^{-\lambda\left(\rho_{k}\right)} \leq r \leq \rho_{k}-\gamma_{k}, \quad k \geq k_{0},
$$

where

$$
\lambda(r)=\frac{\log \Phi\left(\frac{1}{2} r\right)}{\log 2 r},
$$

which tends to infinity as $r$ tends to infinity by $(2.1),\left\{\rho_{k}\right\}$ is a sequence which grows to infinity very quickly, and $\left\{\gamma_{k}\right\}$ is a sequence decreasing to zero in such a way that

$$
\gamma_{k} \rho_{k}^{\lambda\left(\rho_{k}\right)} \rightarrow 0 \quad \text { as } k \rightarrow \infty .
$$

Thus increasing $k_{0}$ if necessary we ensure that all the intervals (4.2) belong to $E_{2}$.

We show that we can assume $\lambda(r)$ increasing. In fact, since $\lambda(r) \rightarrow \infty$ as $r \rightarrow \infty$ there exists $r_{N}$ such that for $r \geq r_{N}, \lambda(r) \geq N$, then we define $\lambda_{1}(r)$ by

$$
\lambda_{1}(r)=N, \quad r_{N} \leq r<r_{N+1},
$$


and $\Phi_{1}(r)$ by the relation

$$
\lambda_{1}(r)=\frac{\log \Phi_{1}\left(\frac{1}{2} r\right)}{\log 2 r} .
$$

Then it is clear that $\lambda(r) \geq \lambda_{1}(r)$ and therefore by (4.3) and (4.5) $\Phi(r) \geq \Phi_{1}(r)$. Hence it is enough to prove that there exists $f$ satisfying (4.1) in a set satisfying (2.2) with $\Phi_{1}(r)$ instead of $\Phi(r)$.

We shall also assume $\rho_{k} \geq \rho_{0}=2$ and $\lambda\left(\rho_{0}\right) \geq 0$, i.e. $\varphi(1) \geq 1$. Then

$$
\rho_{k}^{-\lambda\left(\rho_{k}\right)} \leq 1, \quad \text { so } \rho_{k}-\rho_{k}^{-\lambda\left(\rho_{k}\right)} \geq \frac{1}{2} \rho_{k} .
$$

Then using (4.3), (4.4), and (4.6) we obtain

$$
\begin{aligned}
\int_{E_{2}} \Phi(r) d r & =\int_{E_{2}}(4 r)^{\lambda(2 r)} d r \geq \sum_{k_{0}} \rho_{k}^{\lambda\left(\rho_{k}\right)} \cdot\left\{\rho_{k}-\gamma_{k}-\left(\rho_{k}-\rho_{k}^{-\lambda\left(\rho_{k}\right)}\right)\right\} \\
& =\sum_{k_{0}} \rho_{k}^{\lambda\left(\rho_{k}\right)} \cdot \rho_{k}^{-\lambda\left(\rho_{k}\right)}\left(1-\gamma_{k} \rho_{k}^{\lambda\left(\rho_{k}\right)}\right)>(1+o(1)) \sum_{k_{0}} 1=\infty
\end{aligned}
$$

Next we proceed to construct the function $f$ and the sequence $\left\{\rho_{k}\right\}$. Once $\left\{\rho_{k}\right\}$ has been defined, we will define $\gamma_{k}=\rho_{k}^{-\left(\lambda\left(\rho_{k}\right)+1\right)}$, and since $\rho_{k} \rightarrow \infty,(4.4)$ is satisfied.

We define $f$ by

$$
f(z)=\sum_{1}^{\infty} b_{n} z^{n}
$$

and proceed to construct the coefficients $b_{n}$ successively.

We set $b_{1}=1$. Now let $\left\{p_{k}\right\}$ be a strictly increasing sequence of positive integers such that $p_{1}=1$. We assume that we have already defined $b_{n}$ for $n \leq p_{k}$ and proceed to construct $b_{n}$ for $p_{k}<n \leq p_{k+1}$.

Simultaneously we shall inductively define the increasing sequence of positive numbers $\left\{\rho_{k}\right\}$ with $\rho_{0}=2$ and tending rapidly to infinity and the increasing sequence $\left\{p_{k}\right\}$.

Let us assume that $\rho_{k}$ and $p_{k+1}$ have already been chosen and let $F_{k}(z)$ be the function defined in Lemma 3.4 with $p=p_{k}$ and

$$
a_{n}=b_{n} \rho_{k}^{n} \text { for } 1 \leq n \leq p_{k} .
$$

Then if $a_{n}$ are the coefficients of $F_{k}(z)$ for all $n$, we define $b_{n}$ by (4.8) for $p_{k}<n \leq p_{k+1}$.

Now we assume that $\rho_{k-1}$ and $p_{k}$ have already been chosen. Then we take $\rho_{k}$ so large that the following conditions are satisfied:

$$
\rho_{k}>40 e B_{k}\left(\frac{2 \rho_{k-1}}{A_{0}}\right)^{p_{k}+1}
$$

where

$$
\begin{gathered}
A_{0}=\frac{\sqrt{2}-1}{2}, \quad B_{k}=\sum_{\nu=1}^{p_{k}}\left|b_{\nu}\right|, \\
\rho_{k}>\frac{16 p_{k}^{2}}{2-\sqrt{2}}
\end{gathered}
$$




$$
\rho_{k}^{\lambda\left(\rho_{k}\right)+1} \geq \frac{p_{k}^{3}}{A_{0}}\left(50 C_{k} \rho_{k}^{P_{k}}\right)^{1000} p_{k}^{4}
$$

where $C_{k}=\sum_{\nu=1}^{p_{k}}\left|b_{\nu}\right|$, which can be done since $\lambda(\rho)$ tends to infinity with $\rho$, and finally

$$
\frac{1}{8000 p_{k}^{3}}\left(\lambda\left(\rho_{k}\right)+1\right) \log \rho_{k}-\log 2>8 p_{k} \log \frac{\rho_{k}}{\delta},
$$

where $\delta$ is a number such that $0<\delta<\frac{1}{2}$, for instance we can take $\delta=\frac{1}{3}$, and again (4.12) can be satisfied if $\rho_{k}$ is sufficiently large since $\lambda(\rho)$ tends to infinity as $\rho$ tends to infinity.

Now we show that if $\rho_{k}$ is chosen so that (4.9)-(4.12) are satisfied we can define $p_{k+1}$ so that the inductive definition of the sequences $\left\{\rho_{k}\right\},\left\{p_{k}\right\}$ is finished and therefore also the definition of $f(z)$ in (4.7), and we check that $f(z)$ constructed in this way satisfies (4.1).

First of all we show that if $\rho_{k}$ satisfies (4.9) then

$$
\left|b_{n}\right|<\left(2 \rho_{k-1}\right)^{-n}, \quad p_{k}<n \leq p_{k+1} .
$$

In fact by (3.56) in Lemma 3.4 and Cauchy's inequality we have

$$
\left|a_{n}\right|<40 e M_{k}\left(\frac{\sqrt{2}-1}{2}\right)^{-n}, \quad p_{k}<n \leq p_{k+1},
$$

where

$$
M_{k}=\sum_{\nu=1}^{p_{k}}\left|b_{\nu}\right| \rho_{k}^{\nu}<\rho_{k}^{p_{k}} \cdot \sum_{\nu=1}^{p_{k}}\left|b_{\nu}\right|
$$

which is bigger than $\rho_{1} \geq 2$.

Writing

$$
A_{0}=\frac{\sqrt{2}-1}{2}, \quad B_{k}=\sum_{\nu=1}^{p_{k}}\left|b_{\nu}\right|,
$$

we deduce from(4.8) and (4.14) for $p_{k}<n \leq p_{k+1}$

$$
\left|b_{n}\right| \leq 40 e \rho_{k}^{p_{k}-n} A_{0}^{-n} B_{k} .
$$

Whence we conclude (4.13) if

$$
\rho_{k}^{n-p_{k}}<40 e\left(\frac{2 \rho_{k-1}}{A_{0}}\right)^{n} B_{k},
$$

i.e.

$$
\rho_{k}>\left(40 e B_{k}\right)^{1 /\left(n-p_{k}\right)} \cdot\left(\frac{2 \rho_{k}-1}{A_{0}}\right)^{n /\left(n-p_{k}\right)},
$$

and this will be satisfied for all $n>p_{k}$ if (4.9) holds since $B_{k} \geq\left|b_{1}\right|=1$.

From (4.13), we deduce that $f(z)$ given by (4.7) is an integral function.

Next we show that all the roots of the equations

$$
F_{k}\left(\frac{z}{\rho_{k}}\right)=0 \text { and } F_{k}\left(\frac{z}{\rho_{k}}\right)=i
$$

are in the disc $\left\{z|| z \mid<\rho_{k}-\rho_{k}^{-\lambda\left(\rho_{k}\right)}\right\}$. 
In fact, let us recall that $F_{k}(z)=F\{\omega(z)\}$, where

$$
\omega(z)=\frac{\sum_{\nu=1}^{p_{k}} a_{\nu} z^{\nu}+\mu z^{2 p_{k}}}{\mu+\sum_{\nu=1}^{p_{k}} \bar{a}_{\nu} z^{2 p_{k}-\nu}},
$$

where $e M_{k}<\mu<10 e M_{k}$ and $M_{k}=\sum_{\nu=1}^{p_{k}}\left|a_{\nu}\right|=\sum_{\nu=1}^{p_{k}}\left|b_{\nu}\right| \rho_{k}^{\nu}$.

The equations $F(\omega)=0$ and $F(\omega)=i$ have at most one root in $\{\omega|| \omega \mid<1\}$. Let $\omega_{0}$ and $\omega_{1}$, respectively, be these roots in case there are any.

The roots of (4.15) are the same as those of

$$
\omega\left(\frac{z}{\rho_{k}}\right)=\omega_{0} \text { and } \omega\left(\frac{z}{\rho_{k}}\right)=\omega_{1} .
$$

Therefore we can deal with (4.16) instead of (4.15).

The equation $\omega(z)=\varsigma$ has precisely $2 p_{k}$ roots for $|\zeta|<1$, in particular the equations $\omega(z)=\omega_{0}$ and $\omega(z)=\omega_{1}$ have in total $4 p_{k}$ roots. Let us write $s=$ $\max \left\{\left|\omega_{0}\right|,\left|\omega_{1}\right|\right\}$; then we show that $s<\sqrt{2}-1$, so that it is less than a number between zero and one independent of $k$. In fact $\omega_{0}=0$ and since $F$ is univalent in $|z|<\sqrt{2}-1$, Koebe's $\frac{1}{4}$-theorem implies $\left|\omega_{1}\right|<\sqrt{2}-1$ provided that $\mu>4$ and since

$$
\mu>e M_{k}>M_{k}=\sum_{\nu=1}^{p_{k}}\left|a_{\nu}\right|=\sum_{\nu=1}^{p_{k}}\left|b_{\nu}\right| \rho_{k}^{\nu}
$$

it will be enough to have $\rho_{1}>4$, which is implied by the assumptions $\rho_{0}=2$ and (4.9). Therefore by the left-hand inequality of (3.50), all the roots of the two equations above must satisfy

$$
1-16 p_{k}^{2}\left(1-\left|\frac{z}{\rho_{k}}\right|\right)<s
$$

i.e.

$$
\left|\frac{z}{\rho_{k}}\right|<1-\frac{1-s}{16 p_{k}^{2}}
$$

We now make use of (4.10) and obtain

$$
\rho_{k}>\frac{16 p_{k}^{2}}{2-\sqrt{2}}=\frac{16 p_{k}^{2}}{1-(\sqrt{2}-1)}>\frac{16 p_{k}^{2}}{1-s} .
$$

From (4.18), we get

$$
1-\frac{1-s}{16 p_{k}^{2}}<1-\frac{1}{\rho_{k}}<\frac{\rho_{k}-\rho_{k}^{-\lambda\left(\rho_{k}\right)}}{\rho_{k}} .
$$

Therefore all the roots of (4.16) are in $\left\{z|| z \mid<\rho_{k}-\rho_{k}^{-\lambda\left(\rho_{k}\right)}\right\}$, and the same happens for the roots of $(4.15)$, which was what we were trying to prove.

Since the set $\left\{z\left|\rho_{k}-\rho_{k}^{-\lambda\left(\rho_{k}\right)} \leq\right| z \mid \leq \rho_{k}-\gamma_{k}\right\}$ is compact and the functions $F_{k}\left(z / \rho_{k}\right)$ and $F_{k}\left(z / \rho_{k}\right)-i$ have no zeros in it, there is $\varepsilon_{k}>0$ such that

$$
\left|F_{k}\left(\frac{z}{\rho_{k}}\right)\right|>\varepsilon_{k} \quad \text { and }\left|F_{k}\left(\frac{z}{\rho_{k}}\right)-i\right|>\varepsilon_{k} .
$$

Finally, we will see that $p_{k+1}$, which has not been determined yet, can be take so that (4.1) holds. 
We write

$$
F_{k}\left(\frac{z}{\rho_{k}}\right)=\sum_{n=1}^{\infty} B_{n} z^{n}
$$

and note that the series is absolutely uniformly convergent in $\left\{z \mid \rho_{k}-\rho_{k}^{-\lambda\left(\rho_{k}\right)} \leq\right.$ $\left.|z| \leq \rho_{k}-\gamma_{k}\right\}$, so that we may choose $p_{k+1}$ so large that

$$
\sum_{n=p_{k+1}}^{\infty}\left|B_{n}\right||z|^{n}<\frac{1}{2} \varepsilon_{k}
$$

in this set.

We also have by (4.13)

$$
\sum_{n=p_{k+1}+1}^{\infty}\left|b_{n}\right||z|^{n} \leq \sum_{n=p_{k+1}+1}^{\infty} 2^{-n}=2^{-p_{k+1}}
$$

for the same values of $z$, i.e. taking $p_{k+1}$ large enough we can get that

$$
\sum_{n=p_{k+1}+1}^{\infty}\left|b_{n}\right||z|^{n}<\frac{1}{2} \varepsilon_{k}
$$

And then from (4.21) and (4.22)

$$
\left|f(z)-F_{k}\left(\frac{z}{\rho_{k}}\right)\right|=\left|\sum_{p_{k+1}+1}\left(b_{n}-B_{n}\right) z^{n}\right|<\varepsilon_{k}
$$

in $\left\{z\left|\rho_{k}-\rho_{k}^{-\lambda\left(\rho_{k}\right)} \leq\right| z \mid \leq \rho_{k}-\gamma_{k}\right\}$.

With the choice of $p_{k+1}$ the inductive construction of the sequences $\left\{\rho_{k}\right\}$ and $\left\{p_{k}\right\}$ is complete the therefore also of the function $f$. Furthermore we conclude from (4.20), (4.23), and Rouché's Theorem that the equations

$$
f(z)=0 \text { and } F_{k}\left(\frac{z}{\rho_{k}}\right)=0
$$

have equally many roots in $\{z|| z \mid \leq r\}$ for those values of $r$ in

$$
\rho_{k}-\rho_{k}^{-\lambda\left(\rho_{k}\right)} \leq r \leq \rho_{k}-\gamma_{k}
$$

and the same is true for the equations $f(z)=i$ and $F_{k}\left(z / \rho_{k}\right)=i$.

By Lemma 3.4, the number of the roots of all these equations is at most $2 p_{k}$.

By (4.13) we have $\left|b_{n}\right| \leq 1$ for all $n$. Thus for $0<|z|=\rho<1 / 2$ we have

$$
|f(z)| \leq \sum_{n=1}^{\infty} \rho^{n} \leq \frac{\rho}{1-\rho} \leq 2 \rho<1 .
$$

We also have for such $\rho$

$$
|f(z)| \geq \rho-\sum_{n=2}^{\infty} \rho^{\nu}=\rho-\frac{\rho^{2}}{1-\rho}=\frac{\rho-2 \rho^{2}}{1-\rho}>0 .
$$

Therefore there is $\delta, 0<\delta<\frac{1}{2}$, such that the equations $f(z)=0$ and $f(z)=i$ have no roots different from $z=0$ in $\{z|| z \mid<\delta\}$. 
We deduce from this that for $\rho_{k}-\rho_{k}^{-\lambda\left(\rho_{k}\right)} \leq r \leq \rho_{k}-\gamma_{k}$, we have

$$
n(t, i)=0, \quad t<\delta, \quad \text { and } \quad n(t, i) \leq 2 p_{k}, \quad t \leq r
$$

so that

$$
N(r, i)=\int_{\delta}^{r} \frac{n(t, i)}{t} d t \leq 2 p_{k} \log \frac{r}{\delta}
$$

and since $f(z)$ has a simple zero at the origin and no other zeros in $\{z|0<| z \mid<\delta\}$, we obtain similarly

$$
N(r, 0)=\int_{\delta}^{r} \frac{n(t, 0)}{t} d t+\log \delta \leq 2 p_{k} \log \frac{r}{\delta}
$$

By Lemma 3.4 we have

$$
T\left(r, F_{k}\left(\frac{z}{\rho_{k}}\right)\right) \geq \frac{1}{8000 p_{k}^{3}} \log \frac{1}{1-r / \rho_{k}}
$$

in $\rho_{k}-\rho_{k}^{-\lambda\left(\rho_{k}\right)} \leq r \leq \rho_{k}-\gamma_{k}$ if

$$
\frac{\rho_{k}-\rho_{k}^{-\lambda\left(\rho_{k}\right)}}{\rho_{k}}=1-\rho_{k}^{-\left(\lambda\left(\rho_{k}\right)+1\right)} \geq 1-\frac{A_{0}}{p_{k}^{3}}\left(16 e M_{k}\right)^{-1000 p_{k}^{4}}
$$

where $M_{k}$ is given by $M_{k}=\sum_{\nu=1}^{p_{k}}\left|a_{\nu}\right|$, and by (4.8)

$$
M_{k}=\sum_{\nu=1}^{p_{k}}\left|a_{\nu}\right| \leq \rho_{k}^{p_{k}} \sum_{\nu=1}^{p_{k}}\left|b_{\nu}\right|=C_{k} \rho_{k}^{p_{k}}
$$

where $C_{k}$ depends on the $b_{\nu}, \nu \leq p_{k}$.

Hence (4.27) is satisfied provided that

$$
\rho_{k}^{\lambda\left(\rho_{k}\right)+1} \geq \frac{p_{k}^{3}}{A_{0}}\left(50 C_{k} \rho_{k}^{p_{k}}\right)^{1000 p_{k}^{3}}
$$

which is (4.11).

Therefore (4.26) holds for those $r$ in

$$
\rho_{k}-\rho_{k}^{-\lambda\left(\rho_{k}\right)} \leq r \leq \rho_{k}-\gamma_{k} .
$$

Finally we have by (4.12)

$$
\begin{aligned}
T(r, f(z)) & \geq T\left(r, F_{k}\left(\frac{z}{\rho_{k}}\right)\right)-\log 2 \geq \frac{1}{8000 p_{k}^{3}}\left(\lambda\left(\rho_{k}\right)+1\right) \log \rho_{k}-\log 2 \\
& >8 p_{k} \log \frac{\rho_{k}}{\delta}
\end{aligned}
$$

for $r$ such that

$$
\rho_{k}-\rho_{k}^{-\lambda\left(\rho_{k}\right)} \leq r \leq \rho_{k}-\gamma_{k} .
$$

Thus from (4.24), (4.25), and (4.28) we conclude

$$
N(r, 0)+N(r, i)+N(r, \infty) \leq \frac{1}{2} T(r, f)
$$

in $\rho_{k}-\rho_{k}^{-\lambda\left(\rho_{k}\right)} \leq r \leq \rho_{k}-\gamma_{k}$.

This proves (4.1) and the proof of Theorem 1 is complete. 


\section{BIBLIOGRAPHY}

1. A. Fernandez, Some results about the size of the exceptional set in Nevanlinna's second fundamental theorem, Collect. Math. (to appear).

2. W. K. Hayman, Meromorphic functions, Oxford at the Clarendon Press, 1964.

3. __ Die Nevanlinna-Charakteristik von meromorphen Funktionen und ihren Integralen, Festband zum 70, Geburststag von Rolf Nevanlinna, Springer-Verlag, 1966, pp. 16-20.

4. _ Multivalent functions, Cambridge Univ. Press, 1958.

5. __ On the Valiron deficiencies of integral functions of infinite order, Ark. Mat. 10 (1972), 163-172.

6. J. A. Hempel, Precise bounds in the theorems of Landau and Schottky, Aspects of Contemporary Complex Analysis (D. A. Brannan and J. G. Clunie, eds.), Academic Press, 1980.

7. F. Nevanlinna, Über die Anwendung einer Klasse von uniformisierender Transcendenten zur Untersuchung der Wertverteilung analytischer Funktionen, Acta Math. 50 (1927).

8. R. Nevanlinna, Le théorème de Picard-Borel et la théorie des fonctions méromorphes, GauthierVillars, Paris, 1920.

9. __ Analytic functions, Springer-Verlag, 1970.

Facultad de Matemáticas, Universidad Complutense de Madrid, 28040, MADRID, SPAIN 